\title{
Editorial
}

\section{Interdisciplinary nanophotonics}

https://doi.org/10.1515/nanoph-2019-0236

More than a 100 years ago, Albert Einstein introduced the concept of the photon. It took more than half a century and significant efforts to understand the importance of the concept and the role of this ultra-relativistic (mass rest zero) elementary particle of a bosonic nature (spin 1 , with only two helicity states, \pm 1 ), which can also possess an orbital angular momentum. In regard to quantum information, the nature of the photon is central and the entanglement, after Bell's inequality clarification, promoted the rapid development of quantum optics, quantum information, and quantum computing. At the same time, photon-matter interactions are central in modern nanophotonics. Studying these relies heavily on the ability of a researcher to model and fabricate new structures that involve multiple length scales.

Correspondingly, nanophotonics, in recent years, is clearly showing its inclusive nature. Several disciplines are taking advantages of using novel tools derived from the astonishing progress in nanophotonics for exploring novel fundamental properties of materials on multiple length scales. Optical methods, both experimental and theoretical, are ubiquitous in diverse fields facilitating the development of a common language even for disciplines considered, just few years ago, to be far apart.

The third edition of NANOP, by design, did not have a specific subtitle because the spirit of the conference was aimed at involving a broad range of topics with the aim of bridging the macro world with the molecular world through nanophotonics. This broad approach is representative enough to attract a wide interest in the interdisciplinary communities.

Specifically, we would point out that there has been a noticeable vibrant development of applications in fields such as plasmonics, enhanced spectroscopies, metamaterials, strong light-matter interactions, quantum transport, active nanophotonics (spasers and nanolasers), super-oscillations, nonlinear optical phenomena, quantum aspects, and scattering nanoscopy with surface-enhanced Raman spectroscopy (SERS) applications.

Some of these recent advances in science have produced new technological frontiers with some amazing results: LCD-based mobile phones, nanoparticles for optical tagging and drug delivery in medicine, flexible screens, and wearable devices, just to mention a few.

The NANOP 2018 conference covered a broad list of topics:

- Photonic and plasmonic nanomaterials

- Optical properties of nanostructures

- Optics and transport on two-dimensional (2D) materials

- Nonlinear nano-optics

- Quantum dots and color centers

- Strong light-matter interactions at the nanoscale

- Metamaterials and metasurfaces

- Enhanced spectroscopy and sensing

- Nano-optomechanics

- Quantum nano-optics

- Nanoscale photothermal effects

- Optical sensing form solid state to bio-medicine

For this special issue we accepted a selected list of 12 papers that reflect rather comprehensively, albeit not extensively, the content and the spirit of the 2018 conference. 


\section{Papers in this special issue}

F. Gabelloni et al. "Long-living nonlinear behavior in $\mathrm{CsPbBr} 3$ carrier recombination dynamics"

This paper shows the evidence of a long temperature-dependent cooling rate for the populations of excitons and free carriers. This behavior can be ascribed to a long-living population released by high energy traps, eventually related to the surfaces.

I. Shutsko et al. "Enhanced hybrid optics by growing silver nanoparticles (AgNPs) at local intensity hot spots" This paper shows an experimental proof that growth of AgNPs from the liquid phase at a substrate surface can be controlled by light. Such facile scalable nanofocusing structures could improve the efficiency of plasmonic phenomena in low-cost and potentially large-area applications.

G. Pettinari et al. "Plasmon-assisted bandgap engineering in dilute nitrides"

In this paper, bowtie nanoapertures resonant to the $\mathrm{N}-\mathrm{H}$ complex dissociation energy were numerically modeled by finite element method simulations, implemented by a lithographic approach, and characterized by scanning probe microscopy and resonant scattering spectroscopies. The main result is that the bandgap engineering through plasmonic nanostructures can be optimized for future, efficient realization of site-controlled single photon emitters and for their deterministic integration in plasmonic devices.

R. Yasukuni et al. "Quantitative analysis of SERS spectra of MnSOD over fluctuating aptamer signals using multivariate statistics"

This paper addresses quantitative analysis of SERS spectra of manganese superoxide dismutase (MnSOD) in different concentrations via signals from a specific aptamer using multivariate statistical analysis. The calculated correlation between the SERS spectra and MnSOD concentrations demonstrate that a partial least square regression (PLSR) would be a useful technique for the calibration of protein concentrations from fluctuated SERS spectra of protein-aptamer systems.

M. Passoni et al. "Slow light with an interleaved p-n junction to enhance performance of integrated Mach-Zehnder silicon modulators"

This paper shows a design of band-edge slow light in silicon waveguide gratings and an interleaved p-n junction gratings with the same periodicity. This is used to achieve optimal matching between the electromagnetic field profile and the depletion regions of the $\mathrm{p}-\mathrm{n}$ junction. The resulting modulation efficiency is strongly improved as compared to common modulators based on normal rib waveguides, even in a bandwidth of $20-30 \mathrm{~nm}$ around the band edge.

J. Huang et al. "Femtomolar detection of nucleic acids based on functionalized gold nanoparticles"

This paper shows highly sensitive detection methods with the detection limits between the femto and attomolar range for tDNA. In addition, the specificity of two strategies was also demonstrated with mismatched DNAs. The proposed method provides a simple, fast, sensitive, and specific DNA biosensor which has the potential to be used for point of care tests (POCTs).

V. Caligiuri et al. "Hybridization of epsilon-near-zero (ENZ) modes via resonant tunneling in layered metal/insulator double nanocavities"

The paper presents a study of the hybridization of ENZ resonances in metal/insulator/metal/insulator/metal (MIMIM) nanocavities, obtaining a very large mode splitting reaching $0.477 \mathrm{eV}$ and the $\mathrm{Q}$ factors of the order of 40 in the visible spectral range. The broad tunability of high-quality ENZ resonances together with their strong coupling efficiency makes such MIMIM cavities an ideal platform for exploring light-matter interaction, for example, by integration of quantum emitters in the dielectric layers.

I. M. Palstra et al. "Hybrid cavity-antenna systems for quantum optics outside the cryostat?"

This paper shows design rules for hybrid resonances from a simple analytical model. It is found that hybrids can outperform the plasmonic and cavity constituents in terms of the Purcell factor, and additionally offer the freedom to reach 
any cavity at a similar Purcell factor. For few-emitter strong-coupling, high-speed low-power coherent or incoherent light sources, particle sensing and vibrational spectroscopy, hybrids provide the unique benefit of very high LDOS, tight plasmonic confinement, yet microcavity.

Y.-T. Chen et al. "Charge transfer and electromagnetic enhancement processes revealed in SERS and TERS of CoPc thin film"

This paper shows that cobalt phthalocyanine (CoPc) Raman peaks are mostly enhanced at $636 \mathrm{~nm}$ excitation through a combination of resonant excitation, high electromagnetic (EM) enhancement, and chemical enhancement due to the charge transfer from the metal to the molecule. Whilst at $532 \mathrm{~nm}$ excitation, the SERS and tip-enhanced Raman scattering (TERS) spectra are dominated by photoluminescence, which originates from a photo-induced charge transfer process from the optically excited molecule to the metal.

V. Ardizzone et al. "Emerging 2D materials for room temperature polaritonics"

In this paper, it is shown that transition metal dichalcogenides (TMDs) and low dimensional hybrid organic-inorganic perovskite (PVK) possess the same characteristics related to planar confinement of their excitons: large binding energies, a wide exciton extension, and a high oscillator strength. Also described are their optoelectronic properties and their capability to achieve strong coupling with light, giving particular attention to polariton-polariton interactions.

M. H. Tahersima et al. "Coupling-enhanced dual ITO layer electro-absorption modulator in silicon photonics” This paper experimentally demonstrates a coupling-enhanced electro-absorption modulator by heterogeneously integrating a novel dual-gated indium-tin-oxide (ITO) phase-shifting tunable absorber placed at a silicon directional coupler region. It also demonstrated that the modulator utilizing strong index-change from both real and imaginary parts of active material enables compact and high-performing modulators using semiconductor foundry-near materials.

P. Prete et al. "Nanoscale spectroscopic imaging of GaAs-AlGaAs quantum well tube nanowires: correlating luminescence with nanowire size and inner multi-shell structure"

This paper reports a study of the luminescence and inner structure of GaAs-AlGaAs quantum well tube (QWT) nanowires by low-temperature cathodoluminescence (CL) spectroscopic imaging in combination with scanning transmission electron microscopy (STEM) tomography. This allowed, for the first time, to find a robust correlation between the luminescence properties of these nanowires, their size and inner three-dimensional (3D) structure down to the nanoscale. These findings are important for future monolithic integration of QWT nanowires onto silicon.

\section{Enzo Di Fabrizio}

Professor of Physical Science and Engineering Division King Abdullah University of Science and Technology, Saudi Arabia, SMILEs Lab (Structural Molecular Imaging by Light Enhanced spectroscopies) https://smiles.kaust.edu.sa/ Pages/Home.aspx, Thuwal 23955-6900, Saudi Arabia, e-mail: enzo.difabrizio@kaust.edu.sa

\section{Mark Stockman}

Regents' Professor of Physics and GSU Center for Nano-Optics (CeNO) Director, Department of Physics and Astronomy, Georgia State University (GSU), Atlanta, GA, USA, e-mail: mstockman@gsu.edu 